\title{
PHASE CHANGE WITH VOIDS AND BUBBLES
}

\author{
Francesco Ascione, Michel Frémond \\ Laboratorio Lagrange, \\ Dipartimento di Ingegneria Civile, \\ Università di Roma "Tor Vergata", Italy
}

\begin{abstract}
We investigate phase transition at the macroscopic level. The main novelty of our approach is the possibility of having voids during the phase change. This aspect is described in the model by the mass balance equation which results in a pressure which has a paramount importance. The state variables are the temperature, the strain tensor, and the volume fractions of the two phases (whose evolutions are described by a vectorial equation coming from the principle of virtual power and related to microscopic motion responsible for the phase change). The theory accounts for the main physical phenomenons, for instance, for the cavitation phenomenon and for pressure dependance of the phase change temperature. The scope of the predictive theory is illustrated with uni-dimensional examples.
\end{abstract}

\section{INTRODUCTION}

Bubbles or voids may appear in phase change. In the ice of a frozen pond, it may be seen very small bubbles. In cast iron voids or bubbles are present. In an ice-cream there are also voids which may influence its quality and even its taste. We address this phase change phenomenon at the macroscopic level and derive a predictive theory. We assume there are two phase volume fractions $\beta_{1}$ and $\beta_{2}, \vec{\beta}=\left(\beta_{1}, \beta_{2}\right)$, [2, 4]. The two phases do not fill the whole volume. As for an example, we assume that $\beta_{1}$ is the liquid volume fraction, water for instance, and $\beta_{2}$ is the solid volume fraction, ice for instance. The voids volume fraction is $\epsilon=1-\beta_{1}-\beta_{2}$.

The predictive theory is presented in paragraphs 2 and 3 describing the equations of motion and the constitutive laws. Its properties are investigated in paragraph 4 . The theory accounts for : the cavitation phenomenon where voids appear when pressure becomes negative in a liquid; the importance of the pressure in a mixture of solid and voids, i.e., in a soil. Thus basic properties of soil mechanics are taken into account. In case the densities of solid and liquid are slightly different, the theory accounts for the dependance of the phase change temperature on the pression as it is the case for ice and water.

The last paragraph is devoted to numerical results illustrating these properties. For the sake of stressing on the physical properties we solve 1-D problems. 


\section{THE MASS BALANCE AND EQUATIONS OF MOTION}

We assume, for the sake of simplicity, the same constant density $\rho$ (i.e., the material is incompressible) and the same velocity $\vec{U}=d \vec{u} / d t$ for the two phases ( $\vec{u}$ is the small displacement of the solid phase). The mass balance is

$$
\frac{\partial}{\partial t}\left(\beta_{1}+\beta_{2}\right)+\left(\beta_{1}+\beta_{2}\right) \operatorname{div} \vec{U}=0 .
$$

The equations of motion result from the principle of virtual power. The power of the interior forces is chosen to depend on the regular strain rates $D(\vec{U})(\vec{U}$ is the macroscopic actual velocity and $\left.D_{i, j}(\vec{U})=1 / 2\left(U_{i, j}+U_{j},{ }_{i}\right)\right)$, and also on $d \vec{\beta} / d t$ and $\operatorname{grad}(d \vec{\beta} / d t)$. This latter quantities are clearly related to the microscopic motions which produce the phase change. The gradient of the volume fraction is introduced to take into account local interactions, i.e., the influence of a material point on its neighbourhood. The virtual power of the interior forces we choose [3], is

$$
\mathcal{P}_{\text {int }}(\vec{V}, \gamma)=-\int_{\Omega} \sigma: D(\vec{V}) d \Omega-\int_{\Omega}(\vec{B} \cdot \vec{\gamma}+H: \operatorname{grad} \vec{\gamma}) d \Omega,
$$

where $\vec{V}, \vec{\gamma}$ are macroscopic and microscopic virtual velocities accounting for microscopic motion. Domain $\Omega$ with boundary $\partial \Omega$ is occupied by the material. Two new nonclassical interior forces appear, $\vec{B}$, the interior microscopic vector work, and $H$, the microscopic work flux tensor. The tensor $\sigma$ is the stress tensor. Let us note that the expression of the volume density of virtual power gives the quantities which are to be related by constitutive laws and to be measured in experiments. In some case, the generalized forces and the generalized strain rates to be related are not so easy to identify and in this perspective the choice of the power of the internal forces is an important element of the predictive theory [4]. The virtual powers of the exterior forces is

$$
\mathcal{P}_{\text {ext }}(\vec{V}, \gamma)=\int_{\Omega} \vec{F} \cdot \vec{V} d \Omega+\int_{\partial \Omega} \vec{G} \cdot \vec{V} d \Gamma
$$

where $\vec{F}$ is the macroscopic volume exterior force, $\vec{G}$ the macroscopic surface exterior force. For the sake of simplicity, we assume there is no exterior source of work producing the phase change. We assume that the evolution is quasistatic. Thus the virtual power of acceleration forces is null. The principle of virtual power is

$$
\forall(\vec{V}, \gamma), \mathcal{P}_{\text {ext }}(\vec{V}, \gamma)+\mathcal{P}_{\text {int }}(\vec{V}, \gamma)=0
$$

It gives the equations of motion for the microscopic motions responsible of the evolution of $\beta_{1}$ and $\beta_{2}$ and for the macroscopic motion resulting from the body and surface exterior forces $\vec{F}$ and $\vec{G}$

$$
\begin{aligned}
& -\left(\begin{array}{c}
B_{1} \\
B_{2}
\end{array}\right)+\operatorname{div}\left(\begin{array}{c}
\vec{H}_{1} \\
\vec{H}_{2}
\end{array}\right)=0, \text { in } \Omega,\left(\begin{array}{c}
\vec{H}_{1} \\
\vec{H}_{2}
\end{array}\right) \cdot \vec{N}=0, \text { in } \partial \Omega, \\
& \operatorname{div} \sigma+\vec{F}=0, \text { in } \Omega, \sigma \cdot \vec{N}=\vec{G}, \text { in } \partial \Omega,
\end{aligned}
$$

where works $B_{1}, B_{2}$, work flux vectors $\vec{H}_{1}, \vec{H}_{2}$ and stress $\sigma$ are the interior forces. 


\section{THE ENERGY OR ENTROPY BALANCE}

The energy balance or better the equivalent entropy balance [3] is

$$
\begin{gathered}
\frac{d s}{d t}+\operatorname{div} \vec{Q}=d+R, \text { in } \Omega \\
\vec{Q} \cdot \vec{N}=\pi, \text { in } \partial \Omega,
\end{gathered}
$$

where $s$ is the entropy, $\vec{Q}$ the entropy flux vector, $R$ is the volume entropy source, $\pi$ is the entropy intake $(T \vec{Q}$ where $T$ is the temperature, is the heat flux vector, $T R$ is the volume heat source and $T \pi$ is the heat intake) and $d$ is the dissipation,

$d=\frac{1}{T}\left(\left(\sigma-\frac{\partial \Psi}{\partial \epsilon}\right): D(\vec{U})+\left(\vec{B}-\frac{\partial \Psi}{\partial \beta}\right) \cdot \frac{d \vec{\beta}}{d t}+\left(H-\frac{\partial \Psi}{\partial \operatorname{grad} \beta}\right): \operatorname{grad} \frac{d \vec{\beta}}{d t}-\vec{Q} \cdot \operatorname{grad} T\right)$,

where $\Psi$ is the free energy and $\epsilon$ are the small deformations.

\section{THE CONSTITUTIVE LAWS}

The volume free energy we choose is

$$
\Psi(T, \epsilon, \vec{\beta}, \operatorname{grad} \vec{\beta})=\sum_{j=1}^{2} \beta_{j} \Psi_{j}+I_{K}(\beta)+\frac{k}{2}|\operatorname{grad} \vec{\beta}|^{2},
$$

where $\Psi_{j}$ is the free energies of phase ${ }_{j}, I_{K}$ is the indicator function [5] of the convex set of the possible mixtures

$$
K=\left\{\left(\beta_{1}, \beta_{2}\right) \mid 0 \leq \beta_{1} \leq 1,0 \leq \beta_{2} \leq 1, \beta_{1}+\beta_{2} \in[0,1]\right\} .
$$

The term $I_{K}(\vec{\beta})+(k / 2)|\operatorname{grad} \vec{\beta}|^{2}$ is an interaction or mixture free energy. Parameter $k$ characterizes the intensity of the interaction. The effect of $I_{K}(\vec{\beta})$ is to guarantee that the fractions $\beta_{1}$ and $\beta_{2}$ take admissible physical values.

Let us note that even if the free energy of the voids phase is 0 , the voids phase has physical properties due to the mixture free energy which depends on the gradients of $\beta_{1}$ and of $\beta_{2}$. The gradients are related to the interfaces properties: $\operatorname{grad} \beta_{1}$ describes properties of the voids-liquid interface and $\operatorname{grad} \beta_{2}$ describes properties of the voids-solid interface, for instance surface tension. In this setting, the voids have a role in the phase change and make it different from a phase change without voids. The model is simple and schematic but it may be upgraded by introducing sophisticated interaction free energies depending on $\vec{\beta}$ and on $\operatorname{grad} \vec{\beta}$.

Moreover, for the free energies of the phases, we consider the following simplified expressions

$$
\begin{aligned}
& \Psi_{1}(T, \epsilon)=-C T \ln T-\frac{L}{T_{0}}\left(T-T_{0}\right)+\mathcal{L}(T), \\
& \Psi_{2}(T, \epsilon)=-C T \ln T+\frac{1}{2}\left\{\lambda_{e}(\operatorname{tr} \epsilon)^{2}+2 \mu_{e} \epsilon: \epsilon\right\}+\mathcal{L}(T),
\end{aligned}
$$


where $\lambda_{e}$ and $\mu_{e}$ are the elasticity Lamé parameters, $C$ is the heat capacity and $L$ is the latent heat. The linear function $\mathcal{L}(T)$ is defined by

$$
\mathcal{L}(T)=C T_{0} \ln T_{0}+C\left(1+\ln T_{0}\right)\left(T-T_{0}\right) .
$$

It has been chosen in such a way that within the small perturbation assumption when $T-T_{0}=\theta$, with $\theta$ small compared to $T_{0}$, the quantity $-C T \ln T+\mathcal{L}(T)$ is equivalent to its leading term

$$
-C T \ln T+\mathcal{L}(T) \simeq \frac{C}{2 T_{0}}\left(T-T_{0}\right)^{2} .
$$

Remark 1. The free energy of a material is defined up to a linear function of the temperature. The free energies $\Psi_{1}$ and $\Psi_{2}$ are the free energies of the phases of the same material. Then they are defined up to any linear function of $T$ but, of course, this linear function has to the same for $\Psi_{1}$ and $\Psi_{2}$. This linear function, for instance $\mathcal{L}(T)$, has no influence on the thermomechanical properties of the mixture.

Thus the volume free energy is

$$
\begin{aligned}
\Psi(T, \epsilon, \vec{\beta}, \operatorname{grad} \vec{\beta})= & \left(\beta_{1}+\beta_{2}\right)(-C T \ln T+\mathcal{L}(T)) \\
& -\frac{\beta_{1} L}{T_{0}}\left(T-T_{0}\right)+\frac{\beta_{2}}{2}\left\{\lambda_{e}(\operatorname{tr} \epsilon)^{2}+2 \mu_{e} \epsilon: \epsilon\right\} \\
& +I_{K}(\vec{\beta})+\frac{k}{2}|\operatorname{grad} \vec{\beta}|^{2} .
\end{aligned}
$$

For the pseudo-potential of dissipation depending on $g \overrightarrow{r a} d T, \dot{\epsilon}=d \epsilon / d t, d \vec{\beta} / d t$ and on parameters $\chi=T, \beta_{1}, \beta_{2}$, we choose

$$
\begin{aligned}
& \Phi\left(\operatorname{gra} d T, \dot{\epsilon}, \frac{d \vec{\beta}}{d t}, T, \beta_{1}, \beta_{2}\right)= \\
& \frac{\left(\beta_{1}+\beta_{2}\right) \lambda}{2 T}(\operatorname{grad} T)^{2}+\frac{c}{2}\left(\frac{d \vec{\beta}}{d t}\right)^{2}+\frac{\beta_{1}}{2}\left\{\lambda_{v}(t r \dot{\epsilon})^{2}+2 \mu_{v} \dot{\epsilon}: \dot{\epsilon}\right\} \\
& +I_{0}\left(\frac{\partial}{\partial t}\left(\beta_{1}+\beta_{2}\right)+\left(\beta_{1}+\beta_{2}\right) \operatorname{div} \vec{U}\right),
\end{aligned}
$$

where $I_{0}$ is the indicator function of the origin. The viscosity parameters of the fluid are $\lambda_{v}$ and $\mu_{v}$. Viscosity parameter $c$ quantifies the dissipation of the phase change phenomenon or of the microscopic motions involved in the phase change. The last term in (3) is zero if mass balance (1) is satisfied and it is $+\infty$ otherwise. In other words we may say that the presence of the last term in (3) is due to the fact that mass balance (1) is an internal constraint between velocities and it must be included in the expression of the pseudo-potential of dissipation $\Phi$. Let us recall that the pseudo-potential accounts for the properties of the velocities. We will see that this term is related to the pressure in the system. The constitutive laws are given by the derivative of the free energy and 
pseudo-potential of dissipation [3]

$$
\begin{aligned}
& \sigma=\beta_{1}\left(\lambda_{v}(\operatorname{tr} \dot{\epsilon}) \mathbf{1}+2 \mu_{v} \dot{\epsilon}\right)+\beta_{2}\left(\lambda_{e}(\operatorname{tr} \epsilon) \mathbf{1}+2 \mu_{e} \epsilon\right)-\left(\beta_{1}+\beta_{2}\right) p \mathbf{1}, \\
& \left(\begin{array}{c}
B_{1} \\
B_{2}
\end{array}\right) \in\left(\begin{array}{c}
-\frac{L}{T_{0}}\left(T-T_{0}\right) \\
\frac{1}{2}\left\{\lambda_{e}(t r \epsilon)^{2}+2 \mu_{e} \epsilon: \epsilon\right\}
\end{array}\right)+\left(\begin{array}{c}
-C T \ln T+\mathcal{L}(T) \\
-C T \ln T+\mathcal{L}(T)
\end{array}\right) \\
& +\partial I_{K}(\vec{\beta})+c \frac{d \vec{\beta}}{d t}-p\left(\begin{array}{c}
1 \\
1
\end{array}\right), \\
& \vec{H}_{i}=k \operatorname{grad} \beta_{i}, \vec{Q}=-\frac{\lambda\left(\beta_{1}+\beta_{2}\right)}{T} \operatorname{grad} T, \\
& s=\left(\beta_{1}+\beta_{2}\right) C \ln \frac{T}{T_{0}}+\frac{L}{T_{0}} \beta_{1},
\end{aligned}
$$

where $\mathbf{1}$ is the identity matrix, $p$ is the pressure defined by

$$
-p \in \partial I_{0}\left(\frac{\partial}{\partial t}\left(\beta_{1}+\beta_{2}\right)+\left(\beta_{1}+\beta_{2}\right) \operatorname{div} \vec{U}\right)=\mathbb{R} .
$$

Remark 2. The subdifferential set $\partial I_{K}(\vec{\beta})$ of convex set $K$ is the set of the vectors which are normal to triangle $K$ at point $\vec{\beta},[5]$. Note that when $\vec{\beta}$ is interior to $K$, the subdifferential set is $(0,0)$.

This constitutive law implies that the mass balance is satisfied because the subdifferential set $\partial I_{0}\left(\frac{\partial}{\partial t}\left(\beta_{1}+\beta_{2}\right)+\left(\beta_{1}+\beta_{2}\right) d i v \vec{U}\right)$ is not empty. The stress $\sigma$ is an elastic stress in the solid part and a viscous stress in the fluid.

The small perturbation assumption for $T$ and $\beta_{1}+\beta_{2}$ are: $\theta$ is small compare to $T$ and $\left(\beta_{1}+\beta_{2}\right)-\left(\beta_{1}(0)+\beta_{2}(0)\right)$ is small compare to $\left(\beta_{1}(0)+\beta_{2}(0)\right)$. For the sake of simplicity, we assume there is no voids at the initial time or that $\left(\beta_{1}(0)+\beta_{2}(0)\right)-1$ is small. With the small perturbation assumption, the constitutive laws are

$$
\begin{aligned}
& \sigma=\beta_{1}\left(\lambda_{v}(\operatorname{tr} \dot{\epsilon}) \mathbf{1}+2 \mu_{v} \dot{\epsilon}\right)+\beta_{2}\left(\lambda_{e}(\operatorname{tr} \epsilon) \mathbf{1}+2 \mu_{e} \epsilon\right)-p \mathbf{1}, \\
& \left(\begin{array}{c}
B_{1} \\
B_{2}
\end{array}\right) \in\left(\begin{array}{c}
-\frac{L}{T_{0}} \theta \\
0
\end{array}\right)+\partial I_{K}(\vec{\beta})+c \frac{d \vec{\beta}}{d t}-p\left(\begin{array}{c}
1 \\
1
\end{array}\right), \\
& \vec{H}_{i}=k \operatorname{grad} \beta_{i}, \vec{Q}=-\frac{\lambda}{T} g \overrightarrow{r a} d T, \\
& s=C\left(1+\frac{\theta}{T_{0}}\right)+\frac{L}{T_{0}} \beta_{1},
\end{aligned}
$$

where the deformations $\epsilon$ and temperatures $\theta$ at the power 2 have been neglected in agreement with the small deformation assumption.

Remark 3. An other way to have simple constitutive laws is to assume that the voids are filled with vapor with heat capacity $C$ and thermal conductivity $\lambda$. The functions $\Psi$ and $\Phi$ 
become

$$
\begin{aligned}
\Psi(T, \epsilon, \vec{\beta}, \operatorname{grad} \vec{\beta})= & -C T \ln T-\frac{\beta_{1} L}{T_{0}}\left(T-T_{0}\right)+\frac{\beta_{2}}{2}\left\{\lambda_{e}(\operatorname{tr} \epsilon)^{2}+2 \mu_{e} \epsilon: \epsilon\right\} \\
& +I_{K}(\vec{\beta})+\frac{k}{2}|\operatorname{grad} \vec{\beta}|^{2}, \\
\Phi\left(\operatorname{gra} d T, \dot{\epsilon}, \frac{d \vec{\beta}}{d t}, T, \beta_{1}, \beta_{2}\right)= & \frac{\lambda}{2 T}(\operatorname{gra} d T)^{2}+\frac{c}{2}\left(\frac{d \vec{\beta}}{d t}\right)^{2}+\frac{\beta_{1}}{2}\left\{\lambda_{v}(\operatorname{tr} \dot{\epsilon})^{2}+2 \mu_{v} \dot{\epsilon}: \dot{\epsilon}\right\} \\
& +I_{0}\left(\frac{\partial}{\partial t}\left(\beta_{1}+\beta_{2}\right)+\left(\beta_{1}+\beta_{2}\right) d i v \vec{U}\right) .
\end{aligned}
$$

They give the following constitutive laws

$$
\begin{aligned}
\sigma & =\beta_{1}\left(\lambda_{v}(\operatorname{tr} \dot{\epsilon}) \mathbf{1}+2 \mu_{v} \dot{\epsilon}\right)+\beta_{2}\left(\lambda_{e}(\operatorname{tr} \epsilon) \mathbf{1}+2 \mu_{e} \epsilon\right)-\left(\beta_{1}+\beta_{2}\right) p \mathbf{1}, \\
B & \in\left(\begin{array}{c}
-\frac{L}{T_{0}}\left(T-T_{0}\right) \\
\frac{1}{2}\left\{\lambda_{e}(\operatorname{tr} \epsilon)^{2}+2 \mu_{e} \epsilon: \epsilon\right\}
\end{array}\right)+\partial I_{K}(\vec{\beta})+c \frac{d \vec{\beta}}{d t}-p\left(\begin{array}{c}
1 \\
1
\end{array}\right), \\
\vec{H}_{i} & =\operatorname{kgrad} \beta_{i}, \vec{Q}=-\frac{\lambda}{T} g r \overrightarrow{r a} d T, \\
s & =C(1+\ln T)+\frac{L}{T_{0}} \beta_{1} .
\end{aligned}
$$

\section{THE PREDICTIVE THEORY}

The equations of the predictive theory result from the equations of motion, the entropy balance and the constitutive laws. They are

$$
\begin{aligned}
& \frac{C}{T_{0}} \frac{\partial \theta}{\partial t}+\frac{L}{T_{0}} \frac{\partial \beta_{1}}{\partial t}-\frac{\lambda}{T_{0}} \Delta \theta=R, \text { in } \Omega, \\
& c \frac{\partial \vec{\beta}}{\partial t}-k \Delta \vec{\beta}+\partial I_{K}(\vec{\beta})+\left(\begin{array}{c}
-\frac{L}{T_{0}} \theta \\
0
\end{array}\right)-p\left(\begin{array}{c}
1 \\
1
\end{array}\right) \ni 0, \text { in } \Omega, \\
& \operatorname{div}\left(\beta_{1}\left(\lambda_{v}\left(\operatorname{tr} \epsilon\left(\frac{d \vec{u}}{d t}\right)\right) \mathbf{1}+2 \mu_{v} \epsilon\left(\frac{d \vec{u}}{d t}\right)\right)+\beta_{2}\left(\lambda_{e} \operatorname{tr} \epsilon(\vec{u}) 1+2 \mu_{e} \epsilon(\vec{u})\right)\right) \\
& -g r a d p+\vec{F}=0, \text { in } \Omega, \\
& \frac{\partial}{\partial t}\left(\beta_{1}+\beta_{2}\right)+\operatorname{div} \frac{\partial \vec{u}}{\partial t}=0, \text { in } \Omega, \\
& \lambda \frac{\partial \theta}{\partial N}=T_{0} \pi, k \frac{\partial \vec{\beta}}{\partial N}=0, \sigma \vec{N}=\vec{G}, \text { in } \partial \Omega, \\
& \theta(x, 0)=\theta^{0}(x), \vec{\beta}(x, 0)=\vec{\beta}^{0}(x), \vec{u}(x, 0)=\vec{u}^{0}(x), \text { in } \Omega .
\end{aligned}
$$

Let us note that the pressure intervene in both equations for macroscopic and microscopic motions which are coupled. Mathematical results may be found in [1]. 


\subsection{Properties of the predictive theory: the cavitation phenomenon}

Let us assume that there are only water and voids, $0<\beta_{1}<1, \beta_{2}=0$ and interpret equation (4) assuming an homogeneous state. We have

$$
\partial I_{K}\left(\beta_{1}, 0\right)=\left(\begin{array}{c}
0 \\
-\mathrm{Q}
\end{array}\right)
$$

with $Q \geq 0$. Equation (4) is

$$
c\left(\begin{array}{c}
\frac{\partial \beta_{1}}{\partial t} \\
0
\end{array}\right)+\left(\begin{array}{c}
0 \\
-Q
\end{array}\right)+\left(\begin{array}{c}
-\frac{L}{T_{0}} \theta \\
0
\end{array}\right)-p\left(\begin{array}{l}
1 \\
1
\end{array}\right) \ni 0 .
$$

It results

$$
\begin{aligned}
& p=-Q<0, \\
& c \frac{\partial \beta_{1}}{\partial t}=p+\frac{L}{T_{0}} \theta .
\end{aligned}
$$

The pressure is negative in the water. If $p+\left(L / T_{0}\right) \theta<0$,

$$
\frac{\partial \beta_{1}}{\partial t}<0
$$

The voids volume fraction increases and from the mass balance we get

$$
\operatorname{div} \vec{U}>0 .
$$

This is the cavitation phenomenon which results from the pressure becoming negative.

Let us also note that if

$$
p+\frac{L}{T_{0}} \theta \geq 0, \theta \geq 0,
$$

we may have only liquid at an equilibrium: $\beta_{1}=1$ and $\left(\partial \beta_{1} / \partial t\right)=0$. Thus large temperature and large pressure make the liquid to be the only phase present at an equilibrium.

\subsection{Properties of the predictive theory: soil mechanics}

In case there is only solid and voids, $\beta_{1}=0,0<\beta_{2}<1$, the equation (4) becomes

$$
c\left(\begin{array}{c}
0 \\
\frac{\partial \beta_{2}}{\partial t}
\end{array}\right)+\left(\begin{array}{c}
-P \\
0
\end{array}\right)+\left(\begin{array}{c}
-\frac{L}{T_{0}} \theta \\
0
\end{array}\right)-p\left(\begin{array}{l}
1 \\
1
\end{array}\right) \ni 0 .
$$

We get that the voids volume fraction either increases or decreases if the pressure is either low or large. This is a property of soils mechanics.

Let us also note that if

$$
p \geq 0, \theta \leq 0,
$$

we may have only solid at an equilibrium: $\beta_{2}=1$ and $\left(\partial \beta_{2} / \partial t\right)=0$. Thus positive pressure and negative temperature make the solid to be the only phase present at an equilibrium.

We conclude that the pressure $p$ is the main practical tool to govern the voids volume fraction and that the temperature $T$ is the main practical tool to govern the phase change. 
Remark 4. In case we assume the densities of the liquid and solid to be constant but slightly different, the mass balance becomes with $\rho^{0}=\rho_{1} \beta_{1}(0)+\rho_{2} \beta_{2}(0)$

$$
\frac{\partial}{\partial t}\left(\rho_{1} \beta_{1}+\rho_{2} \beta_{2}\right)+\rho^{0} \operatorname{div} \vec{U}=0 .
$$

In case we have no voids and a mixture of ice and water, $\beta_{1}+\beta_{2}=1$, at an equilibrium, we have

with $P \geq 0$. It results that

$$
\partial I_{K}\left(\beta_{1}, \beta_{2}\right)=\left(\begin{array}{c}
P \\
P
\end{array}\right),
$$

$$
\left(\begin{array}{c}
P \\
P
\end{array}\right)+\left(\begin{array}{c}
-\frac{L}{T_{0}} \theta \\
0
\end{array}\right)-p\left(\begin{array}{c}
\frac{\rho_{1}}{\rho^{0}} \\
\frac{\rho_{2}}{\rho^{0}}
\end{array}\right) \ni 0,
$$

or

$$
-\frac{L}{T_{0}} \theta-p\left(\frac{\rho_{1}-\rho_{2}}{\rho^{0}}\right)=0, p \geq 0 .
$$

In this situation the phase change temperature depends on the pressure. It is known that this is the case for the ice-water phase change. Because $\rho_{1}>\rho_{2}$ the phase change temperature decreases when the pressure increases. A consequence of this property is that the ice melts at the bottom of a glacier and lubricates the rock ice contact surface allowing the downhill motion of the glacier.

The previous relationship shows that the pressure is 0 , when the phase change occurs at temperature $0^{\circ} \mathrm{C}$. Thus $p$ is the pressure with respect to the atmospheric pressure. This phenomenon is investigated in the following section where the macroscopic equation of motion is not as sophisticated as it is in this one.

\section{SOME EVOLUTIONS}

We investigate the evolution of a one-dimensional continuous body occupying domain $(-h / 2, h / 2)$. We denote $w(z, t)$, the vertical displacement; $\theta(z, t)=T(z, t)-T_{0}$, the difference between the absolute temperature and phase change temperature (one may think of the Celsius temperature); $\beta_{i}(z, t)$ the phase volume fractions, with $i=1$ for liquid phase volume fraction and $i=2$ for solid phase volume fraction; $p(z, t)$, the pressure. The exterior forces applied to the structure are $F(z, t)$, the vertical body force and $G(t)$, the surface traction on the top of the structure; $R(z, t)$, is the volume entropy source and $\pi(z= \pm h / 2, t)$, are the surface entropy flows. Quantities $R(z, t) T_{0}$ and $\pi(z= \pm h / 2, t) T_{0}$ are the volume and surface heat sources. We assume the solid is fixed on its bottom face.

The equations of the predictive theory have the following explicit form:

$$
\begin{aligned}
& C \frac{\partial \theta}{\partial t}+L \frac{\partial \beta_{1}}{\partial t}-\lambda \frac{\partial \theta^{2}}{\partial z^{2}}=R T_{0} \\
& c \frac{\partial \beta_{1}}{\partial t}-k \frac{\partial^{2} \beta_{1}}{\partial z^{2}}-\frac{L}{T_{0}} \theta+\partial I_{k}\left(\beta_{1}, \beta_{2}\right)_{1}-p=0 \\
& c \frac{\partial \beta_{2}}{\partial t}-k \frac{\partial^{2} \beta_{2}}{\partial z^{2}}+\partial I_{k}\left(\beta_{1}, \beta_{2}\right)_{2}-p=0
\end{aligned}
$$




$$
\begin{aligned}
& \beta_{1}^{0} \frac{\partial^{2} w}{\partial t \partial z}\left(\lambda_{v}+2 \mu_{v}\right)+\beta_{2}^{0} \frac{\partial w}{\partial z}\left(\lambda_{e}+2 \mu_{e}\right)-\left(\beta_{1}^{0}+\beta_{2}^{0}\right) p=H(z) \\
& \left(\beta_{1}+\beta_{2}\right)+\left(\beta_{1}^{0}+\beta_{2}^{0}\right) \frac{\partial w}{\partial z}=\left(\beta_{1}^{0}+\beta_{2}^{0}\right)\left(1+\frac{\partial w_{0}}{\partial z}\right)
\end{aligned}
$$

where $\partial I_{k}\left(\beta_{1}, \beta_{2}\right)_{1}$ and $\partial I_{k}\left(\beta_{1}, \beta_{2}\right)_{2}$ are the components of elements of the subdifferential set of the indicator function of convex set: $K=\left\{\left(\beta_{1}, \beta_{2}\right) \in R^{2}: 0 \leq \beta_{1} \leq 1,0 \leq \beta_{2} \leq\right.$ $\left.1, \beta_{1}, \beta_{2}-1 \geq 0\right\}$, to which the pair $\left(\beta_{1}, \beta_{2}\right)$ must belong (Figure 2$)$.

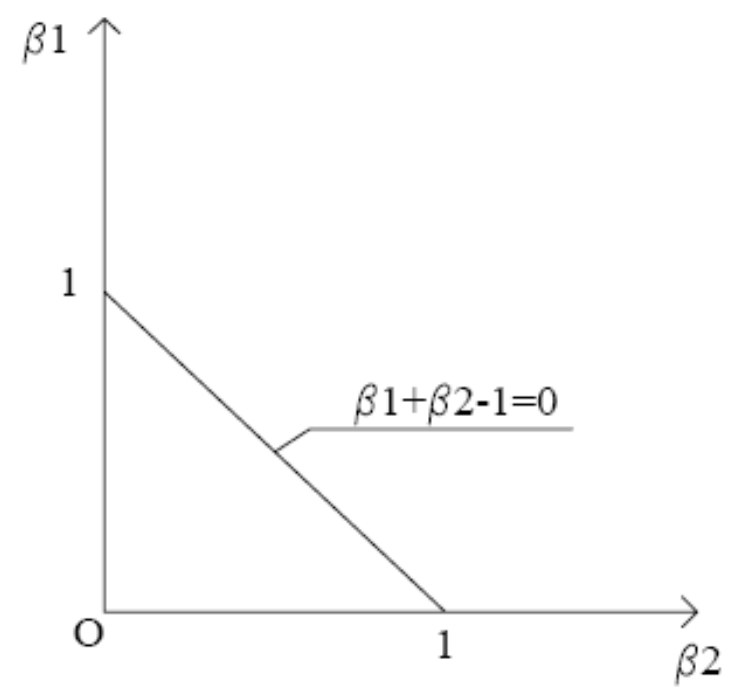

Fig. 1. Convex set K.

Equation (12) results from integration with respect to time of equation (6), while equation (11) results from integration with respect to $\mathrm{z}$ of equation of motion (5). Quantity $H(z)$ is

$$
H(z, t)=G(t)+\int_{\frac{h}{2}}^{z} F(\zeta, t) d \zeta
$$

The set of partial differential equations is completed by: the boundary conditions at the bottom $z=-h / 2$,

$$
\lambda \frac{\partial \theta}{\partial z}=-T_{0} \pi_{l}, \quad k \frac{\partial \beta_{1}}{\partial z}=0, \quad k \frac{\partial \beta_{2}}{\partial z}=0, \quad w=0 ;
$$

the boundary conditions at the top $z=h / 2$,

$$
\lambda \frac{\partial \theta}{\partial z}=T_{0} \pi_{r}, \quad k \frac{\partial \beta_{1}}{\partial z}=0, \quad k \frac{\partial \beta_{2}}{\partial z}=0, \quad \sigma_{z z}=G
$$

and by the initial condition

$$
\theta(z, 0)=\theta^{0}(z), \beta_{1}(z, 0)=\beta_{1}^{0}(z), \quad \beta_{2}(z, 0)=\beta_{2}^{0}(z), w(z, 0)=w^{0}(z),
$$


where $\theta^{0}, \beta_{1}^{0}, \beta_{2}^{0}$ and $w^{0}$ are functions of $\mathrm{z}$.

The evolution problem corresponding to equations (8-12) and the corresponding boundary and initial conditions, is discretized with finite differences. The discredized non linear problem is solved by an iterative method involving a projection on convex set $K[2]$.

We investigate the influence of the physical parameters which intervene in phase change phenomenon: in example 1, the influence of the external load when cavitation occurs; in examples 2 and 3 , the effect of microscopic motion parameter $\mathrm{k}$ which accounts for spatial interactions; in examples 4 and 5 , the influence of the other microscopic motion parameter, c which accounts for the dissipative effects; finally, in example 6 , the influence of pressure p.

For all the aforementioned examples we assume the following values of the boundary conditions:

Boundary condition (bottom $z=-\frac{h}{2}$ )

$$
\lambda \frac{\partial \theta}{\partial z}=0, \quad k \frac{\partial \beta_{1}}{\partial z}=0, \quad k \frac{\partial \beta_{2}}{\partial z}=0, \quad w=0,
$$

Boundary condition (top $z=\frac{h}{2}$ )

$$
\lambda \frac{\partial \theta}{\partial z}=T_{0} \pi_{l}, k \frac{\partial \beta_{1}}{\partial z}=0, k \frac{\partial \beta_{2}}{\partial z}=0, \quad \sigma_{z z}=G .
$$

For what concern the initial conditions, we assume, for all examples, the following values, except in the example 1 (Cavitation) where the volume fraction $\beta_{1}$ and $\beta_{2}$ have the values inverted.

Initial condition

$$
\theta(z, 0)=0, \beta_{1}(z, 0)=0.0, \beta_{2}(z, 0)=1.0, w(z, 0)=0 .
$$

Finally, all other above introduced data assume the values as summarized in table 1.

\section{Table 1. Other common data}

$$
\begin{array}{lll}
\text { geometrical data } & \text { external loads } & \text { mechanical data } \\
h=1.0 \mathrm{~m} & \pi_{l}=-0.5 \frac{W}{m^{2} k} & C=4186000 \frac{\mathrm{J}}{\mathrm{kg} k} \\
t_{f}=8000 \mathrm{~s} & \pi_{l}=-0.5 \frac{W}{m^{2} k} & T_{0}=273.15 \mathrm{k} \\
& \pi_{r}=0.05 \frac{\mathrm{W}^{2} k}{\mathrm{~m}^{2}} & \lambda=2.23 \frac{\mathrm{J}}{\mathrm{s} m} \\
& F=-9180 \mathrm{~Pa} & c=\text { variable } \\
G=\text { variable } & k=\text { variable } \\
& L=335000000 \frac{\mathrm{J}}{\mathrm{m}^{3}} \\
& \mu_{v}=1.82710^{-} \mathrm{Pa} \\
& \lambda_{v}=1.0010^{9} \mathrm{Pas} \\
& \mu_{e}=3.910^{9} \mathrm{~Pa} \\
& \lambda_{e}=10.410^{9} \mathrm{~Pa}
\end{array}
$$

Furthermore the time of observation, $t_{f}$, has a different value, $800000 \mathrm{~s}$ only in examples 1 and 5 . 


\subsection{Example 1: the cavitation phenomenon}

In this subsection we investigate the cavitation phenomenon. It is characterized by an increase of voids volume fraction and a consequent decrease of the water volume fraction due to a low pressure resulting from a tension applied at the top of the structure. The variable parameters $G, c$ and $k$ assume the following values, respectively: $+101000 \mathrm{~Pa},(\mathrm{G}$ is a tension) $10^{7} \mathrm{~J} / \mathrm{m}, 10^{7}(\mathrm{~J} \mathrm{~s}) / \mathrm{m}^{3}$.

The numerical results are reported in figures 2 a-e. In the figures it is shown the evolution of $\theta, \beta_{1}, \beta_{2}, v, w$ and $p$.

a.
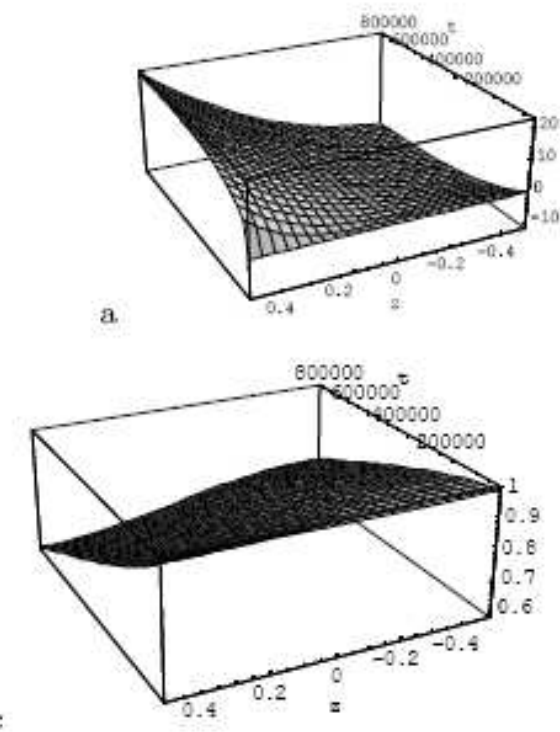

b

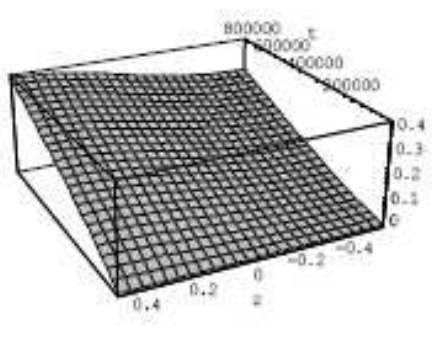

d

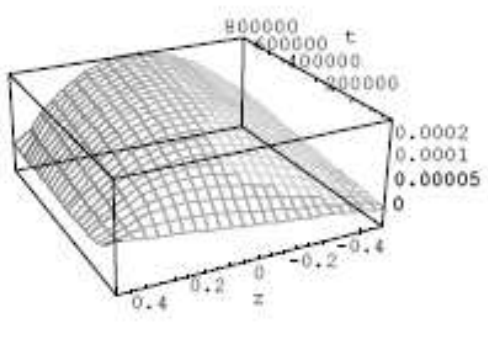

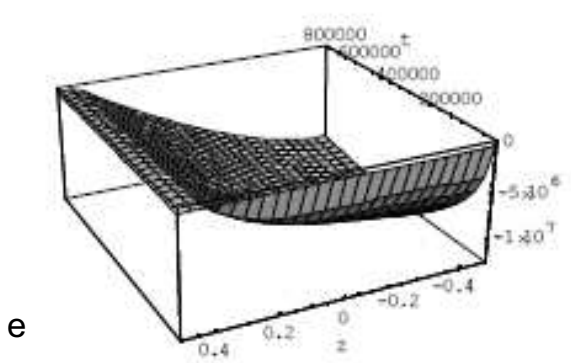

Fig. 2. Example 1 The cavitation phenomenon is due to low pressure $p$ (figure (e)) resulting in creation of voids (figure (b)). Temperature $\theta$, water volume fraction $\beta_{1}$, displacement $w$ are shown in figures (a), (c) and (d).

As expected (see figures 2) in the cavitation phenomenon solid volume fraction remains null. In order to underline the entity of the current phase change, are reported below the values of the voids and water volume fractions at the ends of the structure in correspondence of the final time observation (for all the examples proposed), $t_{f}=800000 \mathrm{~s}$ : at bottom $z=-\frac{h}{2}, \beta_{1}=0.721362, \epsilon=0.278283$, and at top $z=+\frac{h}{2}, \beta_{1}=0.588632$ and 
$\epsilon=0.411954$.

For the sake of simplicity, and because they are the most relevant quantities to describe phase change, only volume fraction $\beta_{1}$ and $\beta_{2}$ are shown in the next examples.

\subsection{Example 2: the non dissipative spatial interaction is important}

In order to see the effect of the interaction parameter $k$, we choose extreme values for this parameter. In this paragraph it is very large, almost equal to $\left.10^{20}(\mathrm{~J} \mathrm{~s}) / \mathrm{m}^{3}\right)$. The other two variable data, c and $G_{z}$ assume the following values, respectively: $10^{7} \mathrm{~J} / \mathrm{m}$, $-101000 \mathrm{~Pa}$.

As already said, we show only volume fractions $\beta_{1}$ and $\beta_{2}$ in Fig. 3 a-b).

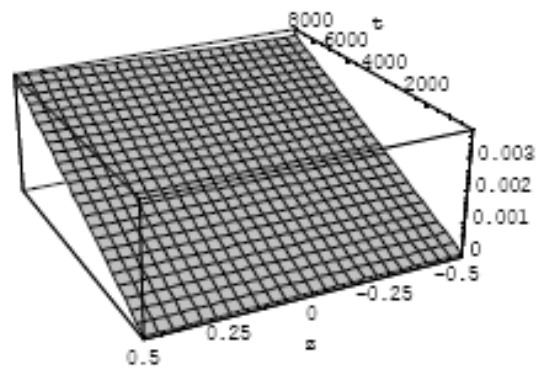

a

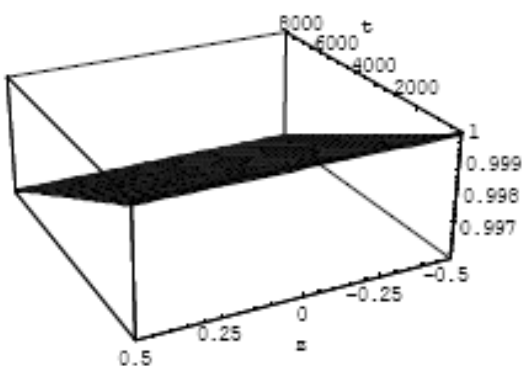

Fig. 3. Example 2 In case there is an important spatial interaction in the phase change, the whole structure is instantaneously affected by the boundary actions. Water volume fraction $\beta_{1}$ and ice volume fraction $\beta_{2}$ are shown in figures (3 a) and $(3 \mathrm{~b})$.

As shown in the figures, when $k$ has a large value, functions $z \longrightarrow \beta(z)$ become constant. For instance, at the both ends of the structure the two volume fractions have the same values:

at bottom $z=-\frac{h}{2}, \beta_{1}=0.003632$ and $\beta_{2}=0.9963$ and at top $z=+\frac{h}{2}, \beta_{1}=0.003728$ and $\beta_{2}=0.996296$.

A large spatial interaction parameter makes the evolution of the $\beta$ 's very dependant of the neighbourhood. On the contrary a very low interaction parameter makes the evolution decooupled and independent of the neighbourhood, as shown in the following paragraph.

\subsection{Example 3: no spatial interaction}

In opposition to previous example 2, parameter k is now almost null: $10^{-20}(\mathrm{~J} s) / \mathrm{m}^{3}$. The other data are those of the previous example. It results that the evolution of the $\beta$ 's are independent of their neighbourhood. Because temperature $\theta$ governing the evolution of the $\beta$ 's, is smooth with respect to space, the $\beta$ 's are also smooth with respect to space. Water and ice volume fractions are shown in figures $4 \mathrm{a}-\mathrm{b}$.

At the two ends of the structure, the volume fractions have independent values: at bottom $z=-\frac{h}{2}, \beta_{1}=0.278283$ and $\beta_{2}=0.721362$, and at top $z=+\frac{h}{2}, \beta_{1}=0.411954$ 
a

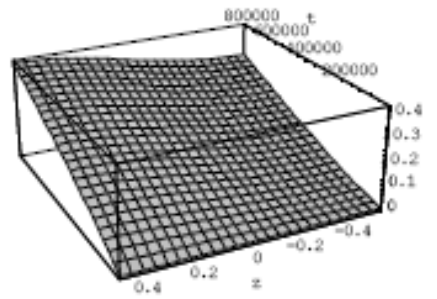

b

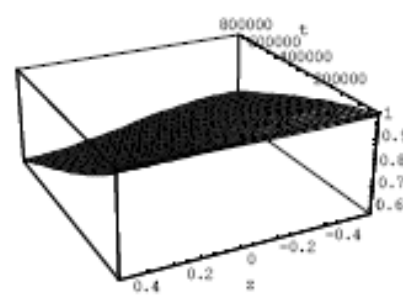

Fig. 4. Example 3 In case there is no spatial interaction in the phase change, the whole structure is not instantaneously affected by the boundary actions. Water volume fraction $\beta_{1}$ and ice volume fraction $\beta_{2}$ are shown in figures (4 a) and (4 b).

and $\beta_{2}=0.588632$.

\subsection{Example 4: dissipative phase change}

In these next two subsections we want to investigate the effect of the dissipative microscopic motion parameter $c$. It controls the velocity of the phase change. For this purpose, we assume firstly it has a very large value: $10^{20} \mathrm{~J} / \mathrm{m}$. The other two variable data, $k$ and $G$ have the following values, respectively: $k=10^{7}(J \mathrm{~s}) / \mathrm{m}^{3}$ and $G_{z}=-101000 \mathrm{~Pa}$. Volume fractions $\beta_{1}$ and $\beta_{2}$ are shown in figures 5 a-b.

a

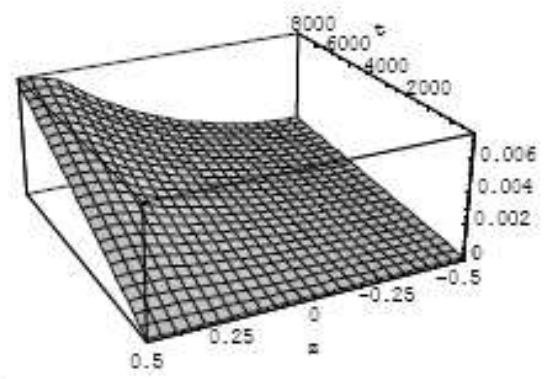

Fig. 5. Example $4 \mathrm{~A}$ dissipative phase change is slow. Water volume fraction $\beta_{1}$ and ice volume fraction $\beta_{2}$ are shown in figures $(5 \mathrm{a})$ and $(5 \mathrm{~b})$.

As expected, functions $t \longrightarrow \beta(t)$ are constant. For instance, we have: at bottom $z=-h / 2, \beta_{1}=0.00154885, \beta_{2}=0.998433$, and at top $z=h / 2, \beta_{1}=$ $0.00711434, \beta_{2}=0.992941$.

\subsection{Example 5: non dissipative phase change}

Contrary to the previous case, we choose $c$ to be almost null with value $10^{-20} \frac{\mathrm{J}}{\mathrm{m}}$, and keep the values of $k$ and $G$ as in example 4 . In agreement with the predictive theory, 
phase change is almost instantaneous as seen in figures (6 a-b). The corresponding volume fractions $\beta_{1}$ and $\beta_{2}$ are: at bottom $z=-h / 2, \beta_{1}=0.278283, \beta_{2}=0.721362$, and at top $z=h / 2, \beta_{1}=0.411954, \beta_{2}=0.588632$.

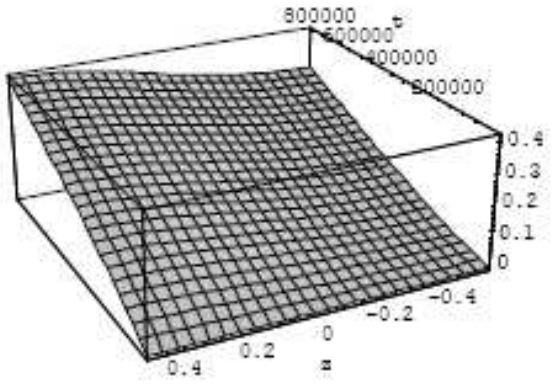

Fig. 6. Example 5 Phase change is rapid in case it is not dissipative. Water volume fraction $\beta_{1}$ and ice volume fraction $\beta_{2}$ are shown in figures (a) and (b).

As c decreases, the velocity of the phase change increases.

\subsection{Example 6: effects of the pressure}

In this last example, we investigate the effect of the pressure $\mathrm{p}$ on the phase change phenomenon. We assume parameters $c$ and $k$ have the following values, respectively: $10^{7} \mathrm{~J} / \mathrm{m}$ and $10^{7}(\mathrm{~J} \mathrm{~s}) / \mathrm{m}^{3}$. We apply a very large compression at the top of the structure with value $G=-1010000 P a$.

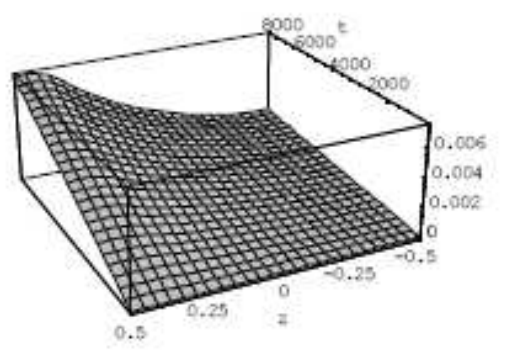

b

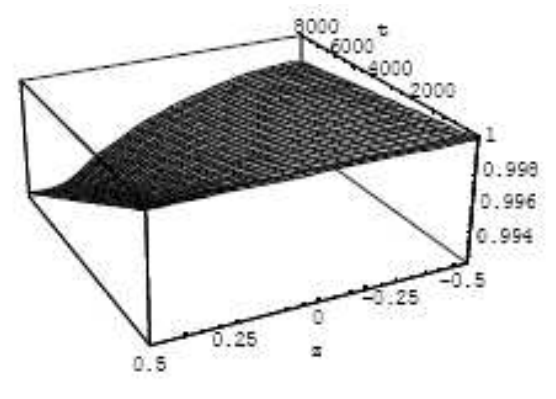

Fig. 7. Example 6 High pressure prevents phase change. Water volume fraction $\beta_{1}$ and ice volume fraction $\beta_{2}$ are shown in figures (a) and (b).

In agreement with the predictive theory, very high pressure prevents phase change as shown in figures $(7 \mathrm{a}-\mathrm{b})$. The volume fractions have the following values at the ends of the structure of figure 5 : at bottom $z=-h / 2, \beta_{1}=0.001500, \beta_{2}=0.998433$, and at top $z=h / 2, \beta_{1}=0.007114, \beta_{2}=0.992941$. 


\section{CONCLUSIONS}

The numerical experiments show that the predictive theory describes the evolution of the different physical quantities in agreement with physics: the pressure has a paramount importance whereas it does not intervene when voids are not taken into account. When the pressure is low cavitation occurs and when it is large it prevents the phase change.

Let us note that the numerical method is effective. The predictive theory which spares the number of parameters to be measured with experiments, has a scope large enough to account for the basic physical features of phase changes involving voids. Of course there are many possible upgrading sophistications in order to give a finer prediction of some aspects of the phenomenons.

\section{REFERENCES}

[1] M. Frémond, E. Rocca: Global existence of solutions for a phase transition model with the possibility of voids, Mathematical Models and Methods in Applied Sciences(M3AS), 4, 16, 2006.

[2] F. Ascione, First results on phase changes with voids, Colloquium Lagrangianum, Scilla, 2006.

[3] M. Frémond, Non-smooth thermomechanics, Springer-Verlag Berlin, 2002.

[4] M. Frémond, Phase change in mechanics, lecture note at the University of Rome Tor Vergata, 2005.

[5] J. J. Moreau, Fonctionnelles convexes, Edizioni del Dipartimento di Ingegneria Civile, dell'Università di Roma Tor Vergata, Roma, ISBN 9788862 960014, 2003.

Received July 13, 2009

\section{SỰ BIẾN ĐỔI PHA TRONG CÁC MÔI TRƯờNG CÓ CHÂN KHÔNG VÀ BONG BÓNG}

Chúng tôi khảo sát sự chuyển pha tại giai đoạn chuyển tiếp ở mức độ vĩ mô. Cái mới của phương pháp tiếp cận là khả năng có chân không trong giai đoạn biến đổi pha. Điều này được mô phỏng bằng phương trình cân bằng khối lượng mà áp suất có tầm quan trọng tối thượng. Các biến trạng thái là nhiệt độ, các tensor biến dạng, và các tỷ lệ khối của hai pha (các khai triển của nó được mô tả bằng một phương trình véc tơ thu được từ các nguyên tắc công khả dĩ và liên quan đến chuyển động vi mô tương ứng với sự thay đổi pha. Lý thuyết giải thích các hiện tượng vật lý chính, chẳng hạn: hiện tượng lỗ trống và sự phụ thuộc áp suất của nhiệt độ làm thay đổi pha. Phạm vi của lý thuyết dự báo được minh họa với các ví dụ một chiều. 